\title{
Prevalence of obesity and diabetes in older people with sarcopenia defined according to EWGSOP2 and FNHI criteria
}

\author{
Francesca Remelli ${ }^{1}$. Elisa Maietti ${ }^{1,14} \cdot$ Pasquale Abete $^{2} \cdot$ Giuseppe Bellelli $^{3} \cdot$ Mario Bo $^{4}$ - Antonio Cherubini ${ }^{5}$. \\ Francesco Corica ${ }^{6} \cdot$ Mauro Di Bari $^{7,8} \cdot$ Marcello Maggio $^{9}$. Maria Rosaria Rizzo ${ }^{10}$. Andrea P. Rossi ${ }^{11}$. \\ Francesco Landi $^{12} \cdot$ Stefano Volpato ${ }^{1,13}$ - the GLISTEN Group Investigators
}

Received: 30 April 2021 / Accepted: 30 July 2021 / Published online: 16 August 2021

(c) The Author(s) 2021

\begin{abstract}
Background Although the prevalence of sarcopenic obesity is increasing, nowadays a universally accepted definition still does not exist. Because, this clinical entity is defined as the combination of obesity and sarcopenia, the diagnosis appears to be strictly linked to criteria used for sarcopenia and the available prevalence data are not uniform. To investigate the prevalence of sarcopenic obesity in older persons according to EWGSOP2 and FNIH criteria. Second, to evaluate the prevalence of diabetes in patients with sarcopenia diagnosed by the two definitions.

Methods Observational multicenter study performed in 2014 on older patients admitted to 12 Italian hospitals (GLISTEN Study). Data were collected through standardized questionnaires, which assessed: socio-demographic data, cognitive status, functional abilities, pharmacological therapy, comorbidities, and blood tests. Moreover, muscle mass and strength and physical performance were evaluated.

Results Six hundred and ten were included in the analyses. Among sarcopenic patients, the prevalence of sarcopenic obesity was $30.8 \%$ with FNIH and 0\% with EWGSOP2 criteria. According to EWGSOP2 criteria, $23.7 \%$ of sarcopenic and $30.8 \%$ of non-sarcopenic patients were affected by diabetes $(p=0.101)$; otherwise, using FNIH criteria, $36.3 \%$ of sarcopenic and $26.9 \%$ of non-sarcopenic patients were diabetic $(p=0.030)$. After adjustment for potential confounders, diabetic patients had a $73 \%$ higher probability of being sarcopenic according to FNIH criteria (OR 1.73; 95\% CI 1.13-2.64).

Conclusions The EWGSOP2 and FNIH sarcopenia criteria are differently related to the prevalence of obesity and diabetes. The EWGSOP2 criteria seem to be not suitable to identify people with sarcopenic obesity.
\end{abstract}

Keywords Sarcopenic obesity $\cdot$ Sarcopenia $\cdot$ Diabetes $\cdot$ Acute care $\cdot$ Mortality

\section{Introduction}

Prevalence of sarcopenic obesity is increasing worldwide, but estimates are not consistent across studies [1,2]. There is not a diagnostic definition of sarcopenic obesity universally accepted and this condition is currently qualified as the co-occurrence of sarcopenia and obesity (i.e., Body Mass Index $\geq 30 \mathrm{Kg} / \mathrm{m}^{2}$ ) [3]: thus, the anthropometric and metabolic characteristics of patients with sarcopenic

The members of the the GLISTEN Group Investigators are listed in appendix.

Stefano Volpato

vlt@unife.it

Extended author information available on the last page of the article obesity might be substantially different according to the diagnostic criteria used for sarcopenia.

Sarcopenia is defined as an age-related decline of skeletal muscle mass with a consequent reduction in muscle strength and physical performance $[4,5]$. The definitions currently used by the scientific community are those proposed by the Working Group on Sarcopenia in Older People (EWGSOP) in 2010 and revised in 2018-EWGSOP2 $[6,7]$ and by the Foundation for the National Institutes of Health (FNIH) Sarcopenia Project [8], that propose different and alternative methods for defining low skeletal muscle mass [9]. Both operational definitions suggest as cut-point for low muscle mass definition an appendicular lean mass (ALM) lower than 20 and $15 \mathrm{~kg}$ for men and women, respectively. Nevertheless, since muscle mass is correlated with body size, ALM can be adjusted for body 
size in different ways, i.e. using height squared (ALM/ height ${ }^{2}$ ), weight (ALM/weight) or body mass index (ALM /BMI). EWGSOP2 consensus recommends using ALM/ height ${ }^{2}<7.0$ and $<5.5 \mathrm{~kg} / \mathrm{m}^{2}$ in men and women, respectively, as cut-off points for low muscle mass definition [7] and FNIH recommendations include an ALM to BMI ratio $(\mathrm{ALM} / \mathrm{BMI})<0.789$ in men and $<0.512$ in women [8].

We hypothesized that different methods for muscle mass standardization might capture different aspects of the sarcopenic phenotype with opposite anthropometric and metabolic characteristics, including but not limited to obesity and type 2 diabetes $[10,11]$, according to the diagnostic criteria used.

The aim of our study was, therefore, to investigate the prevalence of obesity and type 2 diabetes in older persons with sarcopenia defined according to different methods for muscle mass standardization, evaluating the concordance between the two methods.

\section{Methods}

\section{Study design and data collection}

Data were obtained from the Gruppo di Lavoro Italiano Sarcopenia-Trattamento E Nutrizione (GLISTEN) project, a cohort study performed in Geriatrics and Internal Medicine acute care wards of 12 Italian hospitals (Monza, Turin, Ferrara, Verona, Parma, Florence, Ancona, Rome, Napoli I, Napoli II, Cagliari, Messina). Methodology of the GLISTEN project has been described in detail elsewhere [12]. Briefly, the study was designed to investigate the prevalence and clinical correlates of sarcopenia in older hospitalized patients in Italy and to estimate the incidence of sarcopenia during hospital stay. All study centers obtained ethical approval from their institutions; participants signed a written informed consent. All patients consecutively admitted to the participating wards from February 2014 and May 2014 were screened for enrollment. Exclusion criteria were age younger than 65 years and patient's unwillingness to take part in the study. All patients were assessed within 2 days since hospital admission and were followed until discharge. Participants' data were collected through a standardized dedicated questionnaire including demographic characteristics, self-report functional status, cognitive, and mood assessment; medication use; incident and prevalent medical conditions; and biochemical test results. Objective measures of muscle mass (bioimpedance analysis [BIA]) and physical performance (handgrip strength and 4-m usual walking speed) were obtained at hospital admission and before discharge. In this study, 45 of the original 655 enrollees were excluded because of some baseline missing data, leading to a final sample of 610 persons (mean age $80.7 \pm 6.6$ years, male $48.7 \%$ ).

\section{Assessment of sarcopenic obesity}

Sarcopenic obesity was defined as the simultaneous presence of sarcopenia and a BMI $\geq 30 \mathrm{~kg} / \mathrm{m}^{2}$ [13]. Sarcopenia was defined as the presence of low muscle mass and low muscle strength, according to EWGSOP2 [7] and FNIH criteria [8].

Muscle mass was measured by BIA using a Quantum/S Bioelectrical Body Composition Analyzer (Akern Srl, Florence, Italy). Whole-body BIA measurements were taken between the right wrist and ankle with the subject in a supine position, whenever possible. Appendicular Skeletal muscle Mass (ASM), equivalent to ALM, was calculated using the following equation by Sergi and colleagues: ASM $(\mathrm{Kg})=-3.964+\left(0.227 \times\right.$ height $^{2} /$ resist $^{-}$ ance $)+(0.095 \times$ weight $)+(1.384 \times$ sex $)+(0.064 \times$ reactance $)$ where height is measured in centimeters, resistance and reactance in ohms, weight in kilograms; for gender, men $=1$ and women $=0$. ASM was standardized by height squared $\left(\mathrm{ASM} / \mathrm{height}^{2}\right.$ ) and Body Mass Index (ASM/BMI) as suggested by EWGSOP2 and FNIH criteria. Low appendicular muscle mass was classified as ASM/height ${ }^{2}$ less than $7.0 \mathrm{~kg} /$ $\mathrm{m}^{2}$ in men and $6.0 \mathrm{~kg} / \mathrm{m}^{2}$ in women, in line with EWGSOP2 cut-off points [14], and as an ASM/BMI ratio lower than 0.789 in men and 0.512 and women, according to FNIH [15].

Muscle strength was assessed by grip strength (GS), measured using a hand-held dynamometer (JAMAR hand dynamometer Model BK-7498, Fred Sammons Inc., Brookfield, IL). Three trials for each hand were performed, and the highest value of the strongest hand was used in the analyses. GS values below $27 \mathrm{~kg}$ in men and $16 \mathrm{~kg}$ in women were considered as abnormal according to the EWGSOP2 consensus [16]. The corresponding cut-offs for the FNIH criteria were 26 and $16 \mathrm{~kg}$ for men and women, respectively [17].

\section{Assessment of diabetes}

Prevalent diabetes mellitus was defined as self-report of physician diagnosis or antidiabetic medication use. Among undiagnosed diabetic participants, presence of diabetes was identified by fasting plasma glucose level $\geq 126 \mathrm{mg} / \mathrm{dL}$, based on the American Diabetes Association 2003 criteria. Current use of antidiabetic medications (oral antidiabetic agents and insulin) was determined during the baseline interview. 


\section{Covariates}

At hospital admission, a clinical interview was performed to every patient collecting sociodemographic variables, home pharmacological therapy and past medical history; Charlson Comorbidity Index (CCI) [18] was calculated to assess comorbidity burden. Functional status in basic activities of daily living (ADL) and cognitive status were evaluated as reported elsewhere [12]; specifically, difficulty in three or more activities was defined as severe ADL disability [19].

\section{Statistical analysis}

Demographic and clinical features were presented using mean and standard deviation for continuous variables with approximately normal distribution or median and inter-quartile range [IQR] for numerical variables with asymmetric distribution; frequency and percentage were reported for dichotomous variables.

The characteristics of patients were compared according to gender and the presence of sarcopenia and sarcopenic obesity, using Student's $t$-test and Pearson's Chi-squared test for continuous and categorical variables, respectively.

The variables significatively related to the prevalence of sarcopenia were included in a multivariable logistic regression analysis and results were reported as odds ratio and 95\% confidence interval (OR 95\% CI). $P$ value $<0.05$ were considered statistically significant.

Statistical analyses were performed using Software $\mathrm{R}$ 3.5.0 (R Core Team (2020). R: A language and environment for statistical computing. R Foundation for Statistical Computing, Vienna, Austria. URL https://www.R-project.org/.).

\section{Results}

Selected demographic and clinical characteristics of enrolled patients are shown in Table 1, according to the presence of sarcopenia. Participants with sarcopenia diagnosed by EWGSOP2 and FNIH definitions were 190 (29.5\%) and 146 (23.9\%), respectively. According to EWGSOP2 criteria, sarcopenic patients were older and had a lower BMI than participants without sarcopenia; moreover, the prevalence of osteoporosis, weight loss in the previous 6 months, and impaired cognitive function was more common in the sarcopenic subgroup. On the other hand, compared with patients without sarcopenia (FNIH criteria), a greater BMI and a higher prevalence of severe ADL disability, chronic obstructive pulmonary disease, type 2 diabetes, poorer cognitive performance, higher CCI and number of drugs was present among sarcopenic participants.

The prevalence of FNIH defined sarcopenic obesity was $7.4 \%(n=45)$, whereas, no patients were defined as obese in the sarcopenic group based on EWGSOP2 criteria (Fig. 1). Moreover, according to FNIH definition, sarcopenic obesity was more common among women than among men (47.9\% vs $22.4 \%, p=0.003)$.

Overall prevalence of diabetes was $32.8 \%$, with likelihood of having diabetes being related to sarcopenia differently according to diagnostic criteria used. After muscle mass standardization for height ${ }^{2}$ (EWGSOP2 criteria), $23.7 \%$ of sarcopenic and $30.8 \%$ of non-sarcopenic patients were affected by diabetes $(p=0.101)$, whereas, after standardization by BMI (FNIH criteria), the prevalence of diabetes was

Table 1 Selected baseline characteristics according to different definitions

\begin{tabular}{|c|c|c|c|c|c|c|}
\hline & \multicolumn{3}{|l|}{ EWGSOP2 } & \multicolumn{3}{|l|}{ FNIH } \\
\hline & No Sarcopenia & Sarcopenia & $p$ & No Sarcopenia & Sarcopenia & $p$ \\
\hline$N(\%)$ & $471(77.2)$ & $139(\mathbf{2 2 . 8})$ & & $464(75.9)$ & $146(\mathbf{2 3 . 9})$ & \\
\hline Age, mean \pm SD & $80.2 \pm 6.5$ & $82.4 \pm 6.8$ & $<0.001$ & $80.6 \pm 6.6$ & $80.9 \pm 6.7$ & 0.627 \\
\hline Male sex $(\%)$ & 44.2 & 64.0 & $<0.001$ & 42.0 & 67.1 & $<0.001$ \\
\hline $\mathrm{BMI}$, mean $\pm \mathrm{SD}$ & $27.6 \pm 4.9$ & $22.5 \pm 3.1$ & $<0.001$ & $25.8 \pm 4.8$ & $28.2 \pm 5.4$ & $<0.001$ \\
\hline Weight loss (\%) & 39.1 & 56.1 & 0.003 & 44.2 & 41.5 & 0.571 \\
\hline Severe ADL disability (\%) & 21.4 & 30.2 & $<0.001$ & 20.0 & 34.2 & $<0.001$ \\
\hline SPMSQ, median [IQR] & $2[1 ; 3]$ & $2[1 ; 4]$ & 0.001 & $2[1,4]$ & $3[1,4]$ & 0.027 \\
\hline Congestive heart failure (\%) & 16.4 & 19.4 & 0.397 & 16.8 & 17.8 & 0.780 \\
\hline Type 2 diabetes (\%) & 30.8 & 23.7 & 0.101 & 26.9 & 36.3 & $\mathbf{0 . 0 3 0}$ \\
\hline COPD $(\%)$ & 26.5 & 26.6 & 0.869 & 23.7 & 35.6 & 0.004 \\
\hline $\begin{array}{l}\text { Charlson Comorbidity Index, } \\
\text { median [IQR] }\end{array}$ & $3[2,5]$ & $3[2,4]$ & 0.705 & $3[1,4$ & $3[2,5]$ & 0.027 \\
\hline Number of drugs, mean \pm SD & $6.1 \pm 2.9$ & $6.1 \pm 2.7$ & 0.837 & $5.9 \pm 2.9$ & $6.5 \pm 2.7$ & 0.450 \\
\hline
\end{tabular}

$p$ p value, $S D$ standard deviation, $B M I$ body mass index, $A D L$ activity of daily living, $S P M S Q$ Short Portable Mental Status Questionnaire, $I Q R$ interquartile range, $C O P D$ chronic obstructive pulmonary disease 
$36.3 \%$ in sarcopenic subgroup and $26.9 \%$ in non-sarcopenic one $(p=0.030)$ (Fig. 2).

Finally, the strength of the association between the prevalence of diabetes and sarcopenia was assessed by multivariable logistic regression analysis.

In analyses adjusted for age and gender, patients with diabetes had a greater likelihood of being sarcopenic according to FNIH criteria (OR 1.73; 95\% CI 1.13-2.64), whereas diabetes was not associated to sarcopenia prevalence when defined according to EWGSOP2 criteria (OR 0.84; 95\% IC: $0.53-1.31)$. Adjustment for functional status and comorbidity did not modify the relationship between diabetes and sarcopenia (Table 2-Model 3). Additional adjustment for BMI (Table 2-Model 4) significantly attenuated the strength of the association between diabetes and FNIH-defined sarcopenia; conversely, the strength of the association with EWGSOP2 was substantially increased after adjustment for BMI, although without reaching the statistical significance (OR $1.44 ; 95 \%$ CI 0.91-2.27).

\section{Discussion}

Our data confirm the poor concordance between the two current diagnostic criteria of sarcopenia: indeed, the FNIH and the EWGSOP2 criteria identify different groups of patients and, particularly, the EWGSOP2 criteria do not capture people with sarcopenic obesity. Thus, we were able to identify patients with sarcopenic obesity only by using FNIH criteria, finding a prevalence of $7.4 \%$. Because the methods and the cut-off used to evaluate muscle strength and physical performance are equivalent [7, 8], the different population identified by the two criteria are probably due to the different methods used to identify subjects with reduced muscle mass. Thus, while EWGSOP2 criteria standardizes the appendicular muscle mass for the patient's height squared without considering the body weight, the FNIH criteria uses BMI, therefore, including the patient's weight $[7,8]$. Indeed, the two methods used for appendicular muscle mass standardization captured two groups of patients with significantly different BMI and different prevalence of obesity.

This is the first study that investigated the role of EWGSOP2 and FNIH criteria in estimating the prevalence of sarcopenic obesity in hospitalized older patients; our findings based on a multicenter and prospective cohort extended the results of previous reports [20, 21].

The prevalence of diabetes, a metabolic condition related to both obesity and sarcopenia, was also different in sarcopenic patients according to the two diagnostic criteria: using FNHI criteria, diabetes was significantly more common among the sarcopenic group than in the non-sarcopenic counterpart; conversely, patients with sarcopenia diagnosed by EWGSOP2 definitions had

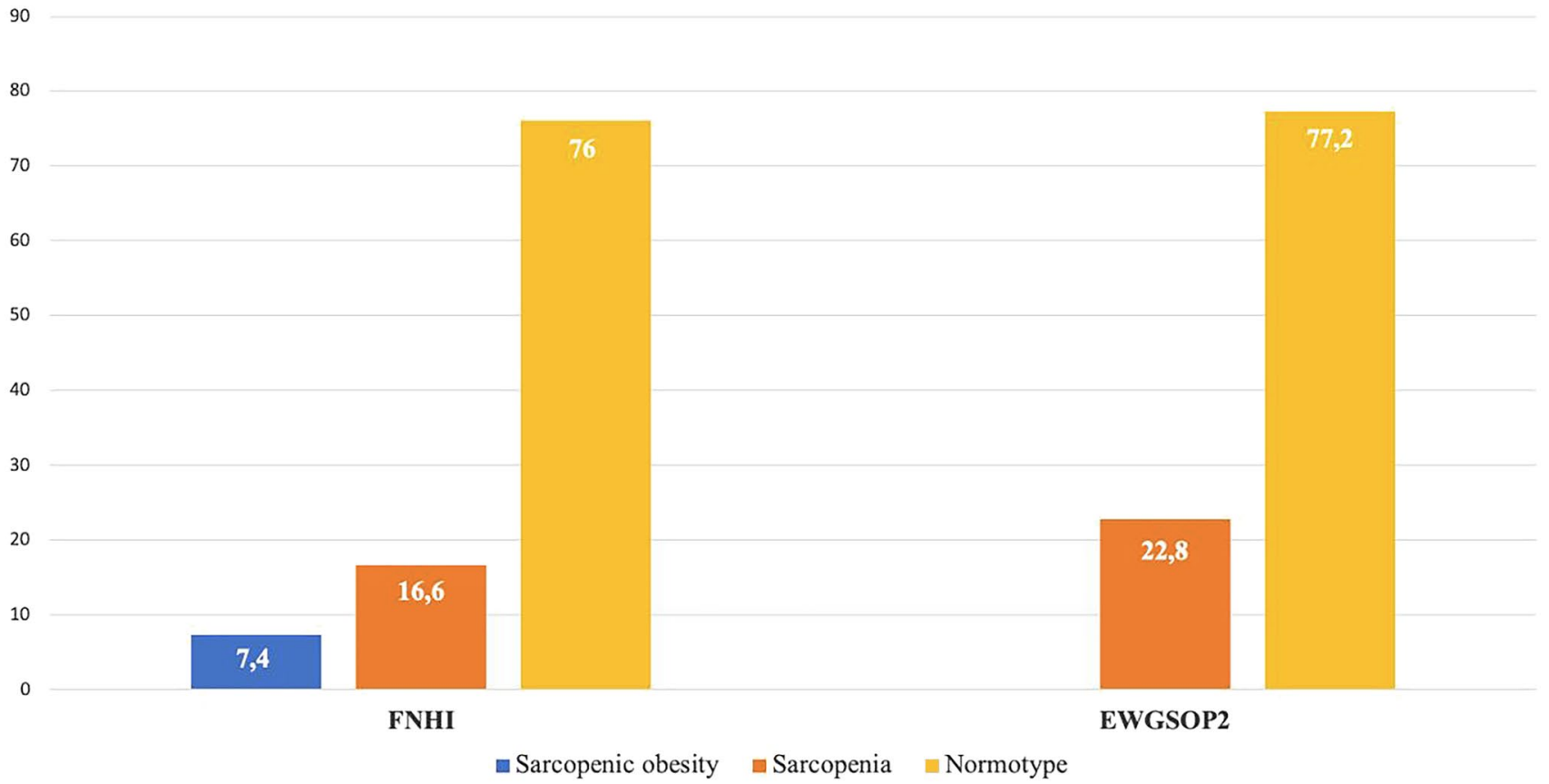

Fig. 1 Prevalence of sarcopenic obesity according to FNIH and EWGSOP2 criteria 


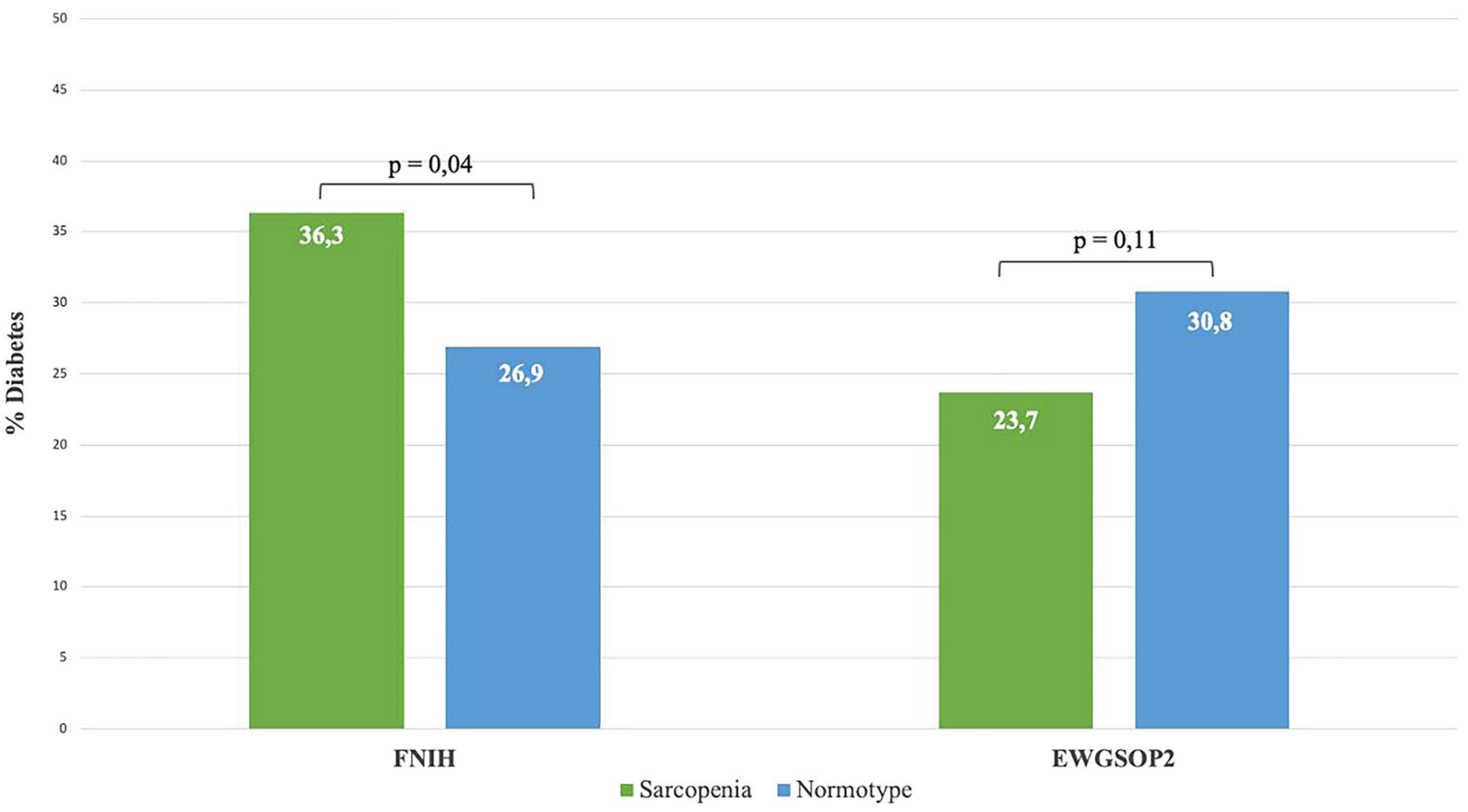

Fig. 2 Prevalence of diabetes related to sarcopenia diagnosed by EWGSOP2 or FNIH criteria

Table 2 Association between diabetes and sarcopenia by FNIH criteria definition adjusted for potential confounders

\begin{tabular}{lllll}
\hline & $\begin{array}{l}\text { Model 1 } \\
\text { OR (95\% CI) }\end{array}$ & $\begin{array}{l}\text { Model 2 } \\
\text { OR } 95 \% \text { CI })\end{array}$ & $\begin{array}{l}\text { Model 3 } \\
\text { OR }(95 \% \text { CI })\end{array}$ & $\begin{array}{l}\text { Model 4 } \\
\text { OR (95\% CI) }\end{array}$ \\
\hline FNHI criteria & & & & \\
Diabetes & $1.55(1.04-2.29)$ & $1.73(1.13-2.64)$ & $1.58(1.04-2.41)$ & $1.33(0.87-2.04)$ \\
Age (years) & - & $1.03(0.68-1.57)$ & $1.02(0.67-1.54)$ & $1.04(0.68-1.58)$ \\
Gender (M) & - & $2.94(1.93-4.46)$ & $3.16(2.08-4.81)$ & $3.88(2.53-5.93)$ \\
BADL & - & - & $1.17(0.77-1.78)$ & $1.16(0.76-1.77)$ \\
SPMSQ & - & - & $0.96(0.63-1.46)$ & $0.97(0.63-1.48)$ \\
Charlson Index & - & - & $1.05(0.69-1.59)$ & $1.02(0.67-1.57)$ \\
BMI & - & - & - & $1.13(0.74-1.73)$ \\
EWGSOP2 criteria & & & & \\
Diabetes & $0.70(0.45-1.08)$ & $0.84(0.53-1.31)$ & $0.79(0.50-1.25)$ & $1.44(0.91-2.27)$ \\
Age (years) & - & $1.07(0.68-1.67)$ & $1.05(0.67-1.67)$ & $1.04(0.66-1.65)$ \\
Gender (M) & - & $2.65(1.69-4.16)$ & $2.73(1.72-4.31)$ & $4.73(2.98-7.49)$ \\
BADL & - & - & $1.10(0.70-1.74)$ & $1.17(0.74-1.85)$ \\
SPMSQ & - & - & $0.98(0.62-1.54)$ & $0.98(0.62-1.55)$ \\
Charlson Index & - & - & $1.02(0.64-1.61)$ & $1.09(0.69-1.73)$ \\
BMI & - & - & - & $0.68(0.43-1.07)$ \\
\hline
\end{tabular}

Model 1 unadjusted, Model 2 adjusted for age and gender, Model 3 adjusted for age, gender, Basic Activity of Daily Living, Short Portable Mental Status Questionnaire and Charlson Index, Model 4 adjusted for age, gender, Basic Activity of Daily Living, Short Portable Mental Status Questionnaire, Charlson Index and Body Mass Index, $O R$ odds ratio, $C I$ confidence interval a lower prevalence of diabetes compared to non-sarcopenic patients $[22,23]$. These results might be certainly explained by the different anthropometric characteristics of EWGSOP2-defined sarcopenic patients because these criteria tend to select underweight patients excluding those with obesity, a condition strongly associated with diabetes [24-26]. Moreover, several studies have demonstrated the presence of pathophysiological mechanisms linking 
diabetes to sarcopenia, including but not limited to the reduced muscle protein synthesis with increased protein catabolism caused by the direct effect of insulin resistance on muscle fibers or the reduction in moto-neurons due to diabetic peripherical neuropathy that leads to muscle atrophy [10, 27-29].

Likewise, in our study the association between diabetes and sarcopenia was confirmed also by multivariable logistic regression analysis, adjusting for potential confounders: diabetes was associated with a higher likelihood of having sarcopenia if assessed by FNIH criteria, while no correlation was demonstrated using EWGSOP2 criteria. Furthermore, in the final model adjusted for BMI, the association between diabetes and FNIH-defined sarcopenia was no longer significant reinforcing the role of obesity as a potential confounder. Using EWGSOP2 criteria, multivariable logistic regression analyses demonstrated an inverse relationship between diabetes and the likelihood of being sarcopenic; nevertheless after further adjustment for BMI the association became direct, with diabetic patients having almost a 50\% increase likelihood of being sarcopenic, although the estimate did not reach the statistical significance. Overall, these finding support the hypothesis that body mass should be included into operational definitions of low muscle mass [30].

In interpreting these findings some limitations should be considered. First, the diagnosis of sarcopenic obesity was simply made considering the presence of sarcopenia in subjects with $\mathrm{BMI} \geq 30$. Second, the assessment of sarcopenia was performed in acutely ill patients, with possible transient impairment in muscle strength, unrelated to sarcopenia. Third, the study was conducted on a sample of hospitalized patient, thus these results have limited external validity.

\section{Conclusions}

The method used for appendicular muscle mass standardization significantly affects the association of sarcopenia with obesity and type 2 diabetes, with standardization for height ${ }^{2}$ (EWGSOP2 criteria) having very low sensitivity for intercepting patients with sarcopenic obesity. Previous studies had already shown that EWGSOP2 and FNIH criteria have different construct and identify different patients: therefore, it is well known that they cannot be used interchangeably $[20,21,31,32]$. Our findings add to this body of evidence, with a specific reference to the diagnosis of sarcopenic obesity and its relationship with diabetes. Further research is needed to examine consistency of sarcopenia definitions to overcome the discrepancy between current diagnostic criteria.

\section{Appendix}

\section{Glisten study group investigators}

Department of Medical Science, University of Ferrara, Ferrara, Italy: Gloria Brombo, Beatrice Ortolani, Elisabetta Savino, Elisa Maietti. Department of Clinical and Experimental Medicine, Geriatric Rehabilitation Department, University of Parma, Parma, Italy: Alberto Fisichella, Valeria Buttò. Department of Medicine, Section of Geriatrics, University of Verona, Verona, Italy: Mauro Zamboni, Cesare Caliari, Elena Ferrari. Research Unit of Medicine of Aging, Department of Experimental and Clinical Medicine, University of Florence, Florence, Italy. Geriatric Intensive Care Unit, Department of Geriatrics and Medicine, Azienda Ospedaliero-Univesitaria Careggi, Florence, Italy: Francesco Orso, Flavia Sacco, Maria Laura Di Meo. Department of Health Sciences, University of Milano Bicocca, Milano, Italy. Acute Geriatric Unit, S. Gerardo Hospital, Monza, Italy: Anna Paola Cerri, Marco Motta, Francesca Pittella, Alessandra Bonfanti. Department of Clinical and Experimental Medicine, University of Messina, Messina, Italy: Sergio Fusco, Roberto Schepisi, Christian Ferro, Antonino Catalano, Stefano Caruso, Luca Soraci. Dipartimento di Scienze Mediche, SCDU Geriatria e Malattie Metaboliche dell'Osso, Città della Salute e della Scienza, Molinette, Torino, Italy: Lorenzo Marchese, Luca Agosta. Department of Translational Medical Sciences, University of Naples Federico II, Naples, Italy: Claudia Basile. Dipartimento di Scienze Mediche, Chirurgiche, Neurologiche, Metaboliche e dell'Invecchiamento, Seconda Università di Napoli, Napoli, Italia: Carla Coppola, Anna Maria Dalise, Ilaria Fava. UOC di Geriatria Ospedale SS. Trinità ASL 8 Cagliari: Olga Catte, Maura Orru', Paolo Salaris. Department of Geriatrics, Neurosciences and Orthopaedics, Catholic University of the Sacred Heart, Rome, Italy: Anna Maria Martone, Elena Ortolani, Sara Salini. Geriatrics and Geriatrics Emergency Care, Italian National Research Center on Aging (IRCCSINRCA), Ancona, Italy: Giuseppina dell'Aquila, Barbara Carrieri.

Author contribution Conceptualization: FR, EM, PA, GB, MB, AC, FC, MDB, MM, MRR, APR, FL, and SV; writing and original draft preparation: FR; writing, review and editing: PA, GB, MB, AC, FC, MDB, MM, MRR, APR, FL, and SV; tables: FR, and EM with edits from SV; visualization/figure: FR, EM, and SV.

Funding Open access funding provided by Università degli Studi di Ferrara within the CRUI-CARE Agreement. This research did not receive any funding. 
Availability of data and material The datasets of the current study are available from the corresponding author on reasonable request.

\section{Declarations}

Conflict of interest The authors have not conflict of interest.

Ethics approval The study protocol received ethical approval by the Ethics Committee of the University of Ferrara. Written consent is obtained from all participants. The data used in this study were anonymized before its use. We confirm that all methods were carried out in accordance with relevant guidelines and regulations.

Informed consent Informed consent was obtained from all individual participants included in the study.

Open Access This article is licensed under a Creative Commons Attribution 4.0 International License, which permits use, sharing, adaptation, distribution and reproduction in any medium or format, as long as you give appropriate credit to the original author(s) and the source, provide a link to the Creative Commons licence, and indicate if changes were made. The images or other third party material in this article are included in the article's Creative Commons licence, unless indicated otherwise in a credit line to the material. If material is not included in the article's Creative Commons licence and your intended use is not permitted by statutory regulation or exceeds the permitted use, you will need to obtain permission directly from the copyright holder. To view a copy of this licence, visit http://creativecommons.org/licenses/by/4.0/.

\section{References}

1. Batsis JA, Villarel DT (2018) Sarcopenic obesity in older adults: aetiology, epidemiology and treatment strategies. Nat Rev Endocrinol 9:513-537. https://doi.org/10.1038/s41574-018-0062-9

2. Batsis JA, Barre LK, Mackenzie TA et al (2013) Variation in the prevalence of sarcopenia and sarcopenic obesity in older adults associated with different research definitions: dual-energy X-ray absorptiometry data from the National Health and Nutrition Examination Survey 1999-2004. J Am Geriatr Soc 61:974-980. https://doi.org/10.1111/jgs.12260

3. Xie W, Xiao G, Fan Y et al (2021) Sarcopenic obesity: research advances in pathogenesis and diagnostic criteria. Aging Clin Exp Res 33:247-252. https://doi.org/10.1007/s40520-019-01435-9

4. Rosenberg IH (1997) Sarcopenia: origins and clinical relevance. J Nutr 127:990S-991S. https://doi.org/10.1093/jn/127.5.990S

5. Meier NF, Lee D (2020) Physical activity and sarcopenia in older adults. Aging Clin Exp Res 32:1675-1687

6. Mithal A, Wahl DA, Bonjour JP et al (2009) IOF Committee of Scientific Advisors (CSA) Nutrition Working Group. Global vitamin D status and determinants of hypovitaminosis D. Osteoporos Int 20:1807-1820. https://doi.org/10.1007/s00198-009-0954-6

7. Cruz-Jentoft AJ, Bahat G, Bauer J et al (2019) Sarcopenia: revised European consensus on definition and diagnosis. Age Ageing 48:16-31. https://doi.org/10.1093/ageing/afy 169

8. Studenski SA, Peters KW, Alley DE et al (2014) The FNIH sarcopenia project: rationale, study description, conference recommendations, and final estimates. J Gerontol Ser A Biol Sci Med Sci 69:547-558. https://doi.org/10.1093/gerona/glu010

9. Soysal P, Smith L, Isik AT (2020) Validation of population-based cut-offs for low muscle mass and strength. Eur Geriatr Med 11:713-714. https://doi.org/10.1007/s41999-020-00336-9
10. Kim TN, Park MS, Yang SJ et al (2010) Prevalence and determinant factors of sarcopenia in patients with type 2 diabetes: the Korean Sarcopenic Obesity Study (KSOS). Diabetes Care 33:1497-1499. https://doi.org/10.2337/dc09-2310

11. Park SW, Goodpaster BH, Strotmeyer ES et al (2007) Accelerated loss of skeletal muscle strength in older adults with type 2 diabetes: the health, aging and body composition study. Diabetes Care 30:1507-1512. https://doi.org/10.2337/dc06-2537

12. Bianchi L, Abete P, Bellelli G et al (2017) Prevalence and clinical correlates of sarcopenia, identified according to the EWGSOP definition and diagnostic algorithm, in hospitalized older people: the GLISTEN study. J Gerontol A Biol Sci Med Sci 72:1575-1581. https://doi.org/10.1093/gerona/glw343

13. Donini LM, Busetto L, Bauer JM et al (2020) Critical appraisal of definitions and diagnostic criteria for sarcopenic obesity based on a systematic review. Clin Nutr 39:2368-2388. https://doi.org/ 10.1016/j.clnu.2019.11.024

14. Gould H, Brennan SL, Kotowicz MA et al (2014) Total and appendicular lean mass reference ranges for Australian men and women: the Geelong osteoporosis study. Calcif Tissue Int 94:363-372. https://doi.org/10.1007/s00223-013-9830-7

15. Cawthon PM, Peters KW, Shardell MD et al (2014) Cutpoints for low appendicular lean mass that identify older adults with clinically significant weakness. J Gerontol A Biol Sci Med Sci 69:567-575. https://doi.org/10.1093/gerona/glu023

16. Dodds RM, Syddall HE, Cooper R et al (2014) Grip strength across the life course: normative data from twelve British studies. PLoS One 9:e113637. https://doi.org/10.1371/journal.pone.01136 37

17. Alley DE, Shardell MD, Peters KW et al (2014) Grip strength cutpoints for the identification of clinically relevant weakness. J Gerontol A Biol Sci Med Sci 69:559-566. https://doi.org/10.1093/ gerona/glu011

18. Deyo RA, Cherkin DC, Ciol MA (1992) Adapting a clinical comorbidity index for use with ICD-9-CM administrative databases. J Clin Epidemiol 45:613-619. https://doi.org/10.1016/ 0895-4356(92)90133-8

19. Ferrucci L, Guralnik JM, Pahor M et al (1997) Hospital diagnoses, medicare charges, and nursing home admissions in the year when older persons become severely disabled. JAMA 277:728-734. https://doi.org/10.1001/jama.1997.03540330050034

20. Bianchi L, Maietti E, Abete P et al (2020) Comparing EWGSOP2 and FNIH sarcopenia definitions: agreement and 3-year survival prognostic value in older hospitalized adults: the GLISTEN study. J Gerontol A Biol Sci Med Sci 75:1331-1337. https://doi.org/10. 1093/gerona/glz249

21. Yang L, Yao X, Shen J et al (2020) Comparison of revised EWGSOP criteria and four other diagnostic criteria of sarcopenia in Chinese community-dwelling elderly residents. Exp Gerontol 130:110798. https://doi.org/10.1016/j.exger.2019.110798

22. Bianchi L, Volpato $S$ (2016) Muscle dysfunction in type 2 diabetes: a major threat to patient's mobility and independence. Acta Diabetol 53:879-889. https://doi.org/10.1007/s00592-016-0880-y

23. Volpato S, Bianchi L, Lauretani F et al (2012) Role of muscle mass and muscle quality in the association between diabetes and gait speed. Diabetes Care 35:1672-1679. https://doi.org/10.2337/ dc11-2202

24. Al-Goblan AS, Al-Alfi MA, Khan MZ (2014) Mechanism linking diabetes mellitus and obesity. Diabetes Metab Syndr Obes 7:587-591. https://doi.org/10.2147/DMSO.S67400

25. Osinski C, Le Gléau L, Poitou C et al (2021) Type 2 diabetes is associated with impaired jejunal enteroendocrine GLP-1 cell lineage in human obesity. Int J Obes 45:170-183. https://doi.org/ 10.1038/s41366-020-00694-1

26. Cierzniak A, Pawelka D, Kaliszewski K et al (2021) MalodobraMazur M. DNA methylation in adipocytes from visceral and 
subcutaneous adipose tissue influences insulin-signaling gene expression in obese individuals. Int J Obes 45:650-658. https:// doi.org/10.1038/s41366-020-00729-7

27. Alemán-Mateo H, López Teros MT, Ramírez FA et al (2014) Association between insulin resistance and low relative appendicular skeletal muscle mass: evidence from a cohort study in community-dwelling older men and women participants. J Gerontol A Biol Sci Med Sci 69:871-877. https://doi.org/10.1093/ gerona/glt193

28. Tuttle LJ, Sinacore DR, Cade WT et al (2011) Lower physical activity is associated with higher intermuscular adipose tissue in people with type 2 diabetes and peripheral neuropathy. Phys Ther 91:923-930. https://doi.org/10.2522/ptj.20100329

29. Khadra D, Itani L, Tannir H et al (2019) Association between sarcopenic obesity and higher risk of type 2 diabetes in adults: a systematic review and meta-analysis. World J Diabetes 10:311-323. https://doi.org/10.4239/wjd.v10.i5.311

30. Sørensen TIA, Frederiksen P, Heitmann BL (2020) Levels and changes in body mass index decomposed into fat and fat-free mass index: relation to long-term all-cause mortality in the general population. Int J Obes 44:2092-2100. https://doi.org/10.1038/ s41366-020-0613-8

31. Savas S, Taşkıran E, Sarac FZ et al (2020) A cross-sectional study on sarcopenia using EWGSOP1 and EWGSOP2 criteria with regional thresholds and different adjustments in a specific geriatric outpatient clinic. Eur Geriatr Med 11:239-246. https:// doi.org/10.1007/s41999-019-00256-3

32. Pal R, Aggarwal A, Singh T et al (2020) Diagnostic cut-offs, prevalence, and biochemical predictors of sarcopenia in healthy Indian adults: the Sarcopenia-Chandigarh Urban Bone Epidemiological Study (Sarco-CUBES). Eur Geriatr Med 11:725-736. https://doi. org/10.1007/s41999-020-00332-z

Publisher's Note Springer Nature remains neutral with regard to jurisdictional claims in published maps and institutional affiliations.

\section{Authors and Affiliations}

\section{Francesca Remelli ${ }^{1}$ - Elisa Maietti ${ }^{1,14}$. Pasquale Abete ${ }^{2} \cdot$ Giuseppe Bellelli $^{3} \cdot$ Mario Bo $^{4}$ - Antonio Cherubini ${ }^{5}$. Francesco Corica ${ }^{6} \cdot$ Mauro Di Bari $^{7,8} \cdot$ Marcello Maggio $^{9} \cdot$ Maria Rosaria Rizzo $^{10}$. Andrea P. Rossi ${ }^{11}$. Francesco Landi $^{12} \cdot$ Stefano Volpato ${ }^{1,13}$ - the GLISTEN Group Investigators}

1 Department of Medical Science, University of Ferrara, Via Aldo Moro, 9, 44124 Ferrara, Italy

2 Department of Translational Medical Sciences, University of Naples Federico II, Naples, Italy

3 School of Medicine and Surgery, University of Milano-Bicocca, Milan, and Acute Geriatric Unit, San Gerardo Hospital, Monza, Italy

4 Section of Geriatrics, Department of Medical Sciences, University of Turin, Città della Salute e della Scienza-Molinette, Torino, Italy

5 Geriatria, Accettazione Geriatrica e Centro di Ricerca per l'Invecchiamento, , IRCCS-INRCA, Ancona, Italy

6 Department of Clinical and Experimental Medicine, University of Messina, Messina, Italy

7 Department of Experimental and Clinical Medicine, Research Unit of Medicine of Aging, University of Florence, Florence, Italy
8 Geriatric Intensive Care Unit, Department of Geriatrics and Medicine, Azienda Ospedaliero-Universitaria Careggi, Florence, Italy

9 Department Medicine and Surgery, Geriatric Rehabilitation Department, University of Parma, Parma, Italy

10 Department of Advanced Medical and Surgical Sciences, University of Campania Luigi Vanvitelli, Naples, Italy

11 Department of Medicine, Geriatrics Division, University of Verona, Verona, Italy

12 Department of Geriatrics, Neurosciences and Orthopaedics, Catholic University of the Sacred Heart, Rome, Italy

13 Center for Clinical Epidemiology, School of Medicine, University of Ferrara, Ferrara, Italy

14 Department of Biomedical and Neuromotor Sciences, University of Bologna, Bologna, Italy 